\title{
Sistemas Agroflorestais na Amazônia Brasileira: Análise de 25 Anos de Pesquisas
}

\author{
Silvio Brienza Júnior(1), Rosana Quaresma Maneschyy(2), Moisés Mourão Júnior ${ }^{(1)}$, Aderaldo Batista Gazel Filho( ${ }^{(3)}$, \\ Jorge Alberto Gazel Yared( ${ }^{(4)}$, Delman Gonçalves ${ }^{(1)}$ e Michelliny Bentes Gama ${ }^{(5)}$
}

${ }^{(1)}$ Embrapa Amazônia Oriental, Travessa Enéas Pinheiro, s/n. C.P. 48, CEP 66095-100. E-mails: brienza@cpatu.embrapa.br;
mmourao@cpatu.embrapa.br; delman@cpatu.embrapa.br; (2)Universidade Federal do Pará, Rua Augusto Corrêa, 01, Guamá, C.P. 479, CEP
66075-110, Belém-PA. E-mail: romaneschy@hotmail.com; ${ }^{(3)}$ EmbrapaAmapá, Rodovia Juscelino Kubitschek, Km 5, 2600, C.P. 10, CEP 68903-419,
Macapá-AP. E-mail: agazel@uol.com.br; (4)Pesquisador aposentado da Embrapa Amazônia Oriental, Governo do Estado do Pará. E-mail:
jyared@amazon.com; ${ }^{(5)}$ Embrapa Rondônia, BR 364, Km 5,5, C.P. 127, CEP 76815-800, Porto Velho-RO. E-mail: mbgama@cpafro.embrapa.br

Resumo - A pesquisa agroflorestal na Amazônia começou a ser sistematizada no início dos anos 80 por instituições como a Empresa Brasileira de Pesquisa Agropecuária (Embrapa), a Comissão Executiva da Lavoura Cacaueira (Ceplac) e o Instituto Nacional de Pesquisa da Amazônia (Inpa). Avaliar a literatura produzida sobre Sistemas Agroflorestais (SAFs) na Amazônia pode identificar áreas carentes de estudos, agrupar informações para consolidação de conhecimento e contribuir para o planejamento e execução de políticas públicas. O presente documento analisa a literatura sobre SAFs na Amazônia brasileira entre 1980 a 2005, baseado no acervo documental da Embrapa Amazônia Oriental e na Base de Dados da Pesquisa Agropecuária (BDPAWeb). Os trabalhos foram classificados por: ano; instituição responsável; local; e uso do(s) componente(s) arbóreo(s) dentro do sistema. Foram catalogadas 460 referências bibliográficas sobre SAFs na Amazônia brasileira. A análise mostrou que as edições do Congresso Brasileiro de Sistemas Agroflorestais contribuíram para aumentar a produção científica. A pesquisa agroflorestal requer longo tempo para a validação de resultados, e uma forma de encurtar tempo é projetar cenários, via utilização de modelagem. Na bibliografia consultada, observa-se uma lacuna quanto à pesquisa de modelagem de sistemas, e como o seu desenvolvimento pode proporcionar avanços dinâmicos e consistentes na pesquisa agroflorestal.

Termos para indexação: Espécies nativas, agrossilvicultura, literatura, banco de dados.

\section{Agroforestry in the Brazilian Amazon: an Analysis of 25 Years of Research}

\begin{abstract}
Agroforestry research in the Brazilian Amazon began in the eighties by Agricultural Research Brazilian Enterprise (Embrapa), Executive Commission of Cacao Plantation (CEPLAC), and the National Institute of Amazonian Research (Inpa) by the Agronomic Science Research Coordination (CPCA). Evaluation of literature on agroforestry systems can identify lacking areas of information or grouping them into knowledge area. Thus, it is possible to extract lessons to be used for planning and executing public polices. This paper analyzed the literature on agroforestry systems, in the Brazilian Amazon, from 1980 to 2005. The bibliography survey was based on database of Embrapa Eastern Amazon as well as on the Agricultural Research Data Base (BDPAWeb) through the search expression [*Amazon and sist* and agriculture* and agrossi*]. The literature was classified by year, responsible institution, author, local implementation, and use of trees. A total of 460 references were classified. The chronological analysis showed that the Brazilian Congress of Agroforestry System (CBSAF) contributed to increase the scientific production. In general, agroforestry research needs a long time for validation. However, projection of scenarios by modeling could shortening time. We observed a gap in modeling of systems. Therefore, it is necessary to develop this issue seeking to obtain more dynamic and solid progresses in the agroforestry system research.
\end{abstract}

Index terms: Native species, agrisilviculture, literature, data bank.

\section{Introdução}

Nas últimas décadas, a cobertura florestal mundial diminuiu acentuadamente (cerca de 20\%) (BRYANT et al.,
1997), em função de mudanças do uso da terra, tanto para a ampliação de áreas de cultivos como para implantação de novas áreas de pastagens (KAIMOWITZ; ANGELSEN, 1998; VOSTI et al., 2002). O desmatamento contínuo 
ameaça os serviços ambientais globais como sequestro de carbono e biodiversidade, especialmente quando associado ao uso do fogo (COCHRANE; SCHULZE, 1998a, 1998b; SORRENSEN, 2000).

O desmatamento na Amazônia brasileira é monitorado oficialmente pelo Instituto Nacional de Pesquisas Espaciais (Inpe) desde 1988. Em termos de valores acumulados, estima-se um desmatamento equivalente a quase 700 mil $\mathrm{km}^{2}$ ou $17,5 \%$ da área florestada da Amazônia (INPE, 2009) e cerca de 19,8\% dessa área encontram-se abandonados ou sub-utilizados ou, muitas vezes, em estado de degradação (ALMEIDA, 2008).

Essa expressiva porção de área desmatada pode ser reincorporada ao processo produtivo mediante o uso de sistemas produtivos adaptados às condições ambientais da Amazônia. Em geral, essas áreas marginais encontram-se sob pressão ampla e direta da pecuária, agricultura e exploração madeireira predatória, provenientes de políticas expansionistas em décadas anteriores (VOSTI et al., 2002; BARRETO et al., 2006).

Atividades agrícolas apresentam benefícios econômicos e podem aliviar a pobreza. Entretanto, limitações agronômicas referentes a tipos de solo, clima e potencial de tecnificação são questões que devem ser consideradas nas prognoses de sustentabilidade e no dimensionamento dos empreendimentos. O debate centra-se em como manejar áreas de florestas restantes, conjugando os objetivos do desenvolvimento via crescimento econômico agrícola com sustentabilidade ambiental e redução da pobreza (VOSTI et al., 2002).

Há várias tecnologias passíveis de uso com o propósito de recuperar a capacidade produtiva do solo, tais como: preparo de área sem queima (KATO, 1998a, 1998b); enriquecimento de floresta secundária para produção de biomassa (BRIENZA JÚNIOR, 1999); sistema Bragantino (CRAVO et al., 2005); pastejo rotacionado (MOURA CARVALHO; COSTA, 1998); integração lavoura-pecuária (TRECENTI et al., 2008) e sistemas agroflorestais (EMBRAPA AMAZÔNIA ORIENTAL, 2003), entre outros. O uso de sistemas agroflorestais (SAFs) também é uma alternativa sustentável, com possibilidade de auxiliar na redução do desmatamento, uma vez que rompe com o ciclo da agricultura migratória tão comum na região, a qual, em função de períodos de pousio muito curtos para a recuperação dos solos, aumenta a pressão sobre as áreas de floresta primária (SMITH et al., 1998).
O espectro de adaptação dos SAFs (condições ecológicas e socioeconômicas) é amplo e pode resultar numa classificação quanto ao uso dos componentes, como: i) sistemas silviagrícolas; ii) silvipastoris; e iii) agrossilvipastoris. Esta ampla adaptação é corroborada pelo elevado número de espécies e arranjos destas registrados na literatura, o que confere um caráter dinâmico aos sistemas agroflorestais (ÁVILA; MINAE, 1992). O enfoque de diagnóstico e planejamento praticado em diversas experiências promissoras de SAFs parte do pressuposto participativo das comunidades para indicação e seleção de espécies, sem que haja restrição quanto ao número de componentes (ARCOVERDE et al., 2004). Desse modo, o número de arranjos possíveis é muito elevado. Smith et al. (1998) citam 70 espécies utilizadas em SAFs na Amazônia brasileira, dentre espécies perenes e semi-perenes, enquanto que Sá (1989) registrou 57 espécies. Por outro lado, Veiga e Tourrand (2002) citam 14 espécies relacionadas somente a sistemas silvipastoris.

Na Amazônia brasileira existem várias experiências sobre recuperação de áreas alteradas com SAFs, havendo predominância de dois grupos:

Primeiro Grupo - Encontram-se os trabalhos realizados por instituições de pesquisa ou independentes cujas informações são geradas de forma sistematizada e dentro de um rigor científico.

Segundo Grupo - Predominam experiências empíricas realizadas por produtores dos mais variados setores. $\mathrm{E}$, nesse caso, os produtores têm maior interesse no resultado final, e não se preocupam com os meios usados na "experimentação". Devido a isso, as informações geradas são de difícil sistematização, mas relevantes, havendo necessidade de serem reunidas, sistematizadas e analisadas de forma a serem submetidas à validação científica.

A prática de combinar árvores com cultivos agrícolas e/ou com atividade pecuária ocorro há bastante tempo na Amazônia brasileira, como exemplo, agricultores japoneses em Tomé-Açu, PA, e agricultores do projeto Reflorestamento Consorciado eAdensado, em Rondônia (Reca), ambos na década de 1980; e agricultores da Associação de Produtores Alternativos de Ouro Preto do Oeste (APA), também em Rondônia, no início dos anos 90. Por outro lado, a pesquisa agroflorestal na Amazônia começou a ser sistematizada no início dos anos 80 por instituições como a Comissão Executiva 
da Lavoura Cacaueira (Ceplac), a Empresa Brasileira de Pesquisa Agropecuária (Embrapa) via suas unidades localizadas na Amazônia e o Instituto Nacional de Pesquisa da Amazônia (Inpa), por meio da Coordenação de Pesquisa em Ciência Agronômicas (CPCA). Outra iniciativa relevante sobre SAFs na Amazônia, refere-se a atuação da Rede Brasileira Agroflorestal (Rebraf), que trabalhou na capacitação de técnicos e agricultores em meados da década de 90, e lançou a publicação "Manual agroflorestal para a Amazônia" (DUBOIS et al., 1996).

A capacidade de pesquisa das instituições na Amazônia não é suficiente para suprir todas as necessidades da região, em virtude não só do elevado número de assuntos de interesse agroflorestal, como também em função do pequeno número de pesquisadores em comparação com a grandeza territorial da Amazônia. Além desses fatores, deve-se considerar, também, que ensaios sobre SAFs ocupam grandes áreas além de exigirem recursos relativamente elevados para monitoramento.

Do ponto de vista acadêmico, avaliar a literatura produzida sobre o tema SAF na Amazônia pode ajudar a identificar áreas carentes de informações, ou agrupar informações para consolidação de conhecimento. E, por outro lado, a avaliação de experiências de SAFs em andamento também pode contribuir para extrair lições a serem utilizadas no planejamento e execução de políticas públicas.

O presente documento refere-se a uma análise da literatura sobre SAFs na Amazônia brasileira, no período de 1980 a 2005. Buscou-se identificar quais as categorias de SAFs mais estudadas (silviagrícola, silvipastoril e agrossilvipastoril), evolução histórica da produção bibliográfica, assim como espécies mais utilizadas, entre outros.

\section{Material e Métodos}

O levantamento bibliográfico realizado sobre SAFs na Amazônia levou em conta o acervo documental da Embrapa Amazônia Oriental, assim como a Base de Dados da Pesquisa Agropecuária (BDPAWeb) por meio da expressão de busca [*amazonia e sist* e agro* e agrossi*]. O material avaliado foi publicado em periódicos, boletins, anais de eventos e folhetos. Não constou do levantamento do presente trabalho teses de pós-graduação. No total, foram catalogadas 460 referências bibliográficas sobre pesquisas realizadas envolvendo SAFs na Amazônia brasileira de 1980 a 2005. Os trabalhos foram classificados por: ano; instituição responsável pela publicação; local de realização do estudo; e uso(s) do(s) componente(s) arbóreo(s) dentro do sistema.

Deve-se ressalvar que, em termos metodológicos, é possível que o procedimento de busca das referências bibliográficas utilizadas não contemple toda a produção científica até a data estipulada no presente trabalho (2005), uma vez que é preciso que as instituições de pesquisa da região procedam a uma constante atualização da Base de Dados da Pesquisa Agropecuária (BDPA).

A análise dos dados foi baseada no uso de tabelas de frequência considerando a década, o local, os usos e as espécies avaliadas. Valores médios foram testados por meio de técnicas não paramétricas. No caso de tabelas de frequências, com múltiplas respostas ou mesmo atribuições, tal como o uso das espécies ou quais as espécies utilizadas, foram construídas relações de concatenação entre estas respostas. Um mapa conceitual também foi elaborado a partir da frequência de linhas de pesquisa, buscando evidenciar a tipologia das linhas de pesquisa e suas orientações ao longo de todo o período avaliado. Para a confecção do mapa conceitual foi utilizado o programa CmapTools (CAÑAS et al., 2004).

\section{Resultados e Discussão}

\section{Evolução histórica da pesquisa em SAFs}

De 1980 a 2005 foram publicados, em média, 18 trabalhos por ano. Uma avaliação linear da produção científica pode não mostrar sua dinâmica ao longo dos anos. Assim, é preciso fazer uma análise da distribuição das publicações ao longo das décadas. E, essa análise cronológica das publicações demonstrou que a produção científica até 1993 é baixa (Figura 1). A média de publicações na década de 80 foi de três por ano. Nos anos 90, esse número passou para 12 por ano, o que implicou em um aumento de cerca de $300 \%$ e alcançou a de média 52 publicações/ano a partir do ano 2000 (crescimento de cerca de 340\% em relação à década de 90) (Tabela 1). Nota-se que esse considerável incremento no número de trabalhos publicados está associado ao advento dos Congressos Brasileiros de Sistemas Agroflorestais (CBSAFs) realizados em 1994, 1998, 2000, 2002 e 2004. Esse fato pode ser comprovado ao se verificar que nos anos de realização dos CBSAFs o número de publicações/ano é muito superior (teste $U$ de MannWhitney: $\left.U=0,001 ; z_{(U) \text { aj. }}=-3,41 ; p<0,001\right)$ aos anos em que estes não ocorrem (Tabela 1). 


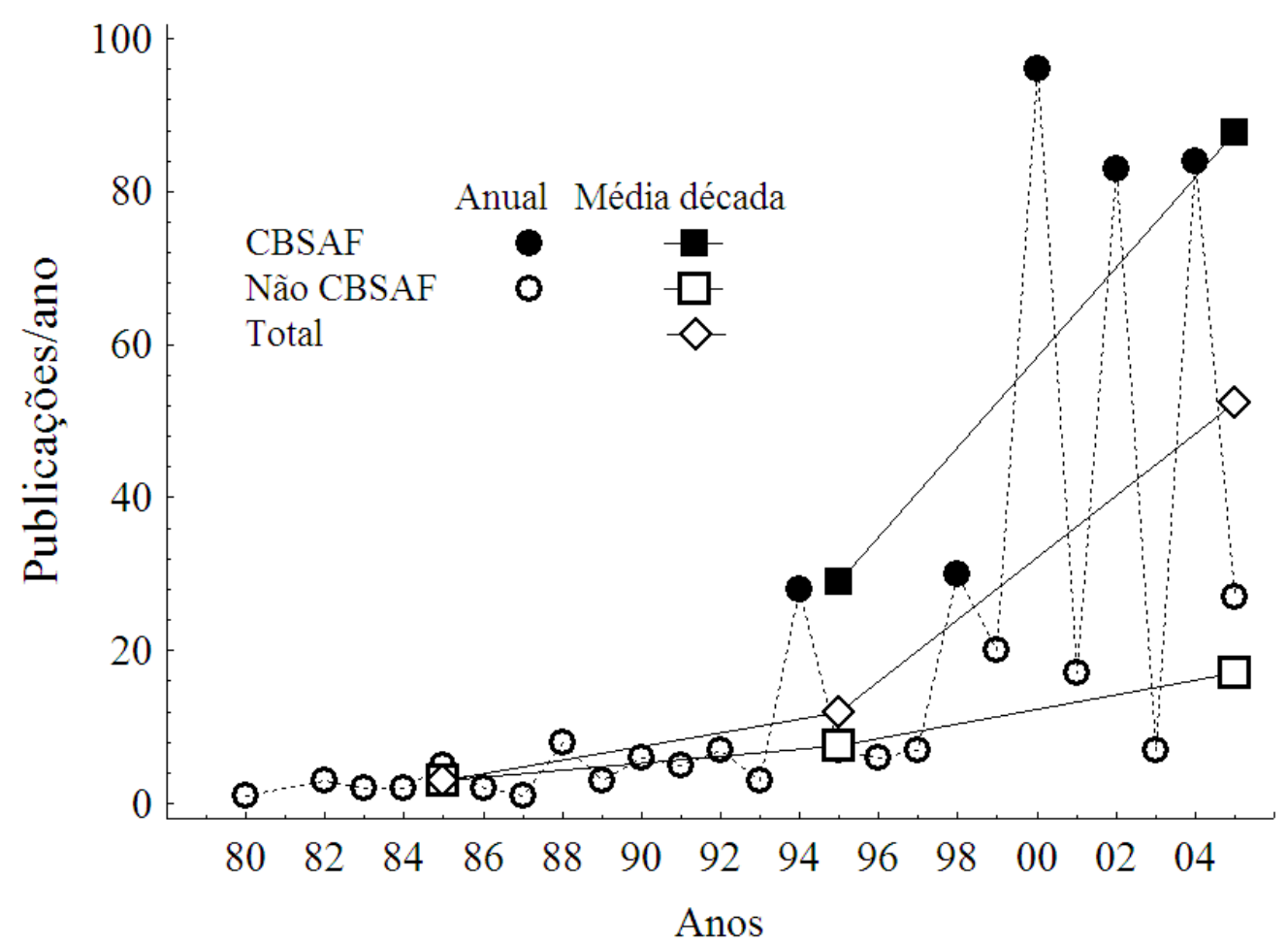

Figura 1. Número de publicações/ano em sistemas agroflorestais na Amazônia brasileira, ao longo de série histórica (1980-2005) e valores médios das décadas, em função da ocorrência dos Congressos Brasileiros de Sistemas Agroflorestais (CBSAFs).

Tabela 1. Número médio de publicações/ano sobre sistemas agroflorestais na Amazônia brasileira nas décadas de 80, 90 e 2000, em função da ocorrência dos Congressos Brasileiros de Sistemas Agroflorestais (CBSAFs).

\begin{tabular}{cccc}
\hline \multirow{2}{*}{ Décadas } & \multicolumn{2}{c}{ Média de publicações por ano } & \multirow{2}{*}{ Média Global } \\
\cline { 2 - 3 } & Sem CBSAF & Com CBSAF & \\
\hline 80 & $3(1-8)^{*}$ & - & $3(1-8)$ \\
90 & $8(3-20)$ & $29(28-30)$ & $12(3-30)$ \\
00 & $17(7-27)$ & $88(83-96)$ & $52(7-96)$ \\
Total & $7(1-27)$ & $64(28-96)$ & $18(1-96)$ \\
\hline
\end{tabular}

* valores entre parêntesis referem-se aos valores mínimo e máximo observados nas décadas.

Considerando-se as décadas de 90 e 2000, verificase um nítido aumento do número de publicações condicionado a realização dos CBSAFs. Por exemplo, na década de 90, o número de publicações/ano com os CBSAFs foi de $88(\mathrm{p}<0,01)$ e de 17 sem os CBSAFs. Esse mesmo comportamento $(\mathrm{p}<0,01)$ também é observado na primeira metade da década de 2000 (Tabela 1 e Figura 1). Deste modo, nota-se uma tendência de centralização das comunicações científicas nos encontros específicos da área agroflorestal.
Diante disso, é visível a contribuição dada pelos encontros específicos da área agroflorestal (CBSAF) para alavancar e divulgar a pesquisa agroflorestal na região. A partir dessa informação, pode-se inferir que existe, na região, uma capacidade instalada para gerar trabalhos científicos que podem subsidiar a formatação de um periódico específico para a área agroflorestal.

\section{Tipologia de SAFs}

Em toda a série histórica consultada, foram registradas pesquisas referentes a todos os tipos de sistemas agroflorestais: silviagrícolas; silvipastoris e agrosilvipastoris (Tabela 2). Observa-se uma maior ênfase da pesquisa em sistemas silviagrícolas (59\% das publicações consultadas), onde $54 \%$ do total dos trabalhos referem-se exclusivamente a sistemas silviagrícolas. A categoria "outros", que abrangeu publicações gerais ou formativas, vem em seguida com cerca de $21 \%$ do total das publicações. Consideradas de forma conjunta, as tipologias de sistemas silvipastoril e agrossilvipastoril somaram $22 \%$ da literatura publicada no período de 1980 a 2005. Publicações abordando mais de uma tipologia foram pouco expressivas, representando somente $5 \%$ do total 
Tabela 2. Número de publicações em função dos focos de ação da pesquisa sobre sistemas agroflorestais na Amazônia brasileira, nas décadas de 80, 90 e 2000.

\begin{tabular}{|c|c|c|c|c|c|c|c|c|c|c|c|}
\hline \multicolumn{4}{|c|}{ Tipos de sistemas agroflorestais } & \multicolumn{6}{|c|}{ Décadas } & \multirow{2}{*}{\multicolumn{2}{|c|}{ Total }} \\
\hline SAG & SSP & ASGSP & Outros & & 80 & 90 & & 00 & & & \\
\hline & & & & & - & - & & 3 & $(1 \%)$ & 3 & $(1 \%)$ \\
\hline & & & {$[+]$} & & - & 21 & $(18 \%)$ & 74 & $(24 \%)$ & 95 & $(21 \%)$ \\
\hline$[+]$ & & & & 20 & $(74 \%)$ & 67 & $(56 \%)$ & 161 & $(51 \%)$ & 248 & $(54 \%)$ \\
\hline \multirow[t]{3}{*}[+]{} & & & {$[+]$} & 1 & $(4 \%)$ & - & & 3 & $(01 \%)$ & 4 & $(1 \%)$ \\
\hline & {$[+]$} & & & 2 & $(7 \%)$ & 8 & $(7 \%)$ & 35 & $(11 \%)$ & 45 & $(10 \%)$ \\
\hline & & {$[+]$} & & 4 & $(15 \%)$ & 20 & $(17 \%)$ & 29 & $(9 \%)$ & 53 & $(12 \%)$ \\
\hline$[+]$ & {$[+]$} & & & & - & 1 & $(1 \%)$ & 2 & $(1 \%)$ & 3 & $(1 \%)$ \\
\hline$[+]$ & & {$[+]$} & & & - & 2 & $(2 \%)$ & 5 & $(2 \%)$ & 7 & $(2 \%)$ \\
\hline$[+]$ & {$[+]$} & {$[+]$} & & & - & - & & 2 & $(1 \%)$ & 2 & $(1 \%)$ \\
\hline \multirow[t]{2}{*}{$\begin{array}{c}264 \\
(59 \%)\end{array}$} & $\begin{array}{c}50 \\
(12 \%)\end{array}$ & $\begin{array}{c}62 \\
(15 \%)\end{array}$ & $\begin{array}{c}99 \\
(22 \%)\end{array}$ & & & & & & & & \\
\hline & \multicolumn{2}{|c|}{ Total } & & \multicolumn{2}{|c|}{$27(100 \%)$} & \multicolumn{2}{|c|}{$119(100 \%)$} & \multicolumn{2}{|c|}{$314(100 \%)$} & $460(1$ & $00 \%)$ \\
\hline
\end{tabular}

Onde: SAG - sistemas silviagrícolas; SSP - sistemas silvipastoris; ASGSP - sistemas agrosilvipastoris; [+] - registro do foco de ação de pesquisa.

Focando-se as tipologias de SAFs consideradas, observa-se que embora tenha sido predominantemente maior, o percentual de trabalhos com sistemas silviagrícolas diminuiu ao longo das décadas. Essa mesma tendência também foi verificada para as tipologias que incluem o componente animal. A categoria "outros" apresentou aumento no percentual de contribuições, sendo que na década de 80 era menor do que $5 \%$, passando a $18 \%$ na década de 90 e chegando a $25 \%$ até a metade da década de 2000 (Tabela 2).

Considerando os locais de desenvolvimento de pesquisas em SAFs, os estados do Pará e Amazonas, juntos, contribuíram com cerca de $65 \%$ dos trabalhos publicados, seguidos dos estados de Rondônia e Acre (20\%), e Roraima e Amapá (7\%). Os estados do Pará e do Amazonas contribuíram desde a década de 80, enquanto que os estados de Roraima e outros pertencentes ao bioma amazônico, tais como Maranhão, Tocantins e
Mato Grosso apresentaram produção científica somente a partir da década de 2000 (Tabela 3).

\section{Usos dos componentes}

No banco de informações pesquisado foi possível identificar diferentes usos atribuídos aos componentes dos SAFs. Considerando o uso principal, uma vez que é possível encontrar mais de um, num mesmo trabalho, o mais frequente foi alimentação $(86 \%)$, seguido de madeira $(52 \%)$, enriquecimento da vegetação $(24 \%)$, forragem $(19 \%)$ e resíduos e óleo (15\%). Com menos de $10 \%$, aparecem os componentes de SAFs para fins de sombreamento (9\%) e uso medicinal (7\%) (Tabela 4). Abaixo de 5\% ficaram os componentes cujos usos foram direcionados para energia (5\%) e fibras $(2 \%)$.

Em termos de uso único, chama a atenção os trabalhos direcionados ao tema segurança alimentar, onde $22 \%$ das publicações são dedicadas a espécies alimentares (Tabela 4). 
Tabela 3. Frequências de publicações sobre sistemas agroflorestais na Amazônia brasileira em função dos locais de estudo de sistemas agroflorestais, nas décadas de 80, 90 e 2000.

\begin{tabular}{lccrr}
\hline & \multicolumn{3}{c}{ Décadas } & \multicolumn{1}{c}{ Total } \\
\cline { 2 - 4 } & $\mathbf{8 0}$ & $\mathbf{9 0}$ & $\mathbf{0 0}$ & \\
\hline Acre & $\sim$ & $7(6 \%)$ & $36(11 \%)$ & $43(9 \%)$ \\
Amapá & $\sim$ & $2(2 \%)$ & $11(4 \%)$ & $13(03 \%)$ \\
Amazonas & $9(33 \%)$ & $40(34 \%)$ & $83(26 \%)$ & $132(29 \%)$ \\
Pará & $12(44 \%)$ & $46(39 \%)$ & $106(34 \%)$ & $164(36 \%)$ \\
Rondônia & $\sim$ & $9(8 \%)$ & $43(14 \%)$ & $52(11 \%)$ \\
Roraima & $\sim$ & $\sim$ & $20(6 \%)$ & $20(4 \%)$ \\
Mato Grosso* & $\sim$ & $\sim$ & $6(2 \%)$ & $6(1 \%)$ \\
Tocantins* & $\sim$ & $\sim$ & $1(\cong 1 \%)$ & $1(\cong 1 \%)$ \\
Maranhão* & $\sim$ & $\sim$ & $\cong$ & $1(\cong 1 \%)$ \\
Não definido & $6(22 \%)$ & $13(11 \%)$ & $6(2 \%)$ & $25(5 \%)$ \\
Outros: Distrito Federal, & $\sim$ & $2(2 \%)$ & $1(\cong 1 \%)$ & $3(\cong 1 \%)$ \\
Rio de Janeiro, São Paulo & $\sim$ & $119(100 \%)$ & $314(100 \%)$ & $460(100 \%)$ \\
\hline Total & $27(100 \%)$ & & & \\
\hline
\end{tabular}

* Estados também pertencentes ao bioma amazônico.

Tabela 4. Usos mais frequentes das espécies componentes de sistemas agroflorestais na Amazônia brasileira ao longo de série histórica (1980-2005).

\begin{tabular}{|c|c|c|c|c|c|c|c|c|}
\hline \multicolumn{9}{|c|}{ Usos dos componentes de SAFs } \\
\hline & $\mathbf{A L}$ & MA & EN & FO & RO & SO & ME & Total ${ }^{*}$ \\
\hline & {$[+]$} & & & & & & & $90(22 \%)$ \\
\hline & {$[+]$} & {$[+]$} & & & & & & $75(19 \%)$ \\
\hline & {$[+]$} & & & & {$[+]$} & & & $29(7 \%)$ \\
\hline & {$[+]$} & {$[+]$} & {$[+]$} & & & & & $26(6 \%)$ \\
\hline & {$[+]$} & {$[+]$} & & {$[+]$} & & & & $25(6 \%)$ \\
\hline & {$[+]$} & & {$[+]$} & & & & & $20(5 \%)$ \\
\hline & & {$[+]$} & & {$[+]$} & & & & $9(2 \%)$ \\
\hline & & & {$[+]$} & & & & & $8(2 \%)$ \\
\hline & {$[+]$} & {$[+]$} & & & & {$[+]$} & & $8(2 \%)$ \\
\hline & {$[+]$} & {$[+]$} & {$[+]$} & {$[+]$} & & & & $8(2 \%)$ \\
\hline & {$[+]$} & {$[+]$} & & & & & {$[+]$} & $7(2 \%)$ \\
\hline & {$[+]$} & {$[+]$} & & & {$[+]$} & & & $5(1 \%)$ \\
\hline & {$[+]$} & {$[+]$} & & & {$[+]$} & & {$[+]$} & $5(1 \%)$ \\
\hline Total & $\begin{array}{c}348 \\
(86 \%)\end{array}$ & $\begin{array}{c}211 \\
(52 \%)\end{array}$ & $\begin{array}{c}99 \\
(24 \%)\end{array}$ & $\begin{array}{c}78 \\
(19 \%)\end{array}$ & $\begin{array}{c}59 \\
(15 \%)\end{array}$ & $\begin{array}{c}37 \\
(9 \%)\end{array}$ & $\begin{array}{c}30 \\
(7 \%)\end{array}$ & $\begin{array}{r}405 \\
(100 \%)\end{array}$ \\
\hline
\end{tabular}

AL - alimento; MA - madeira; EN - enriquecimento; FO - forragem; RO - resíduos e óleos; SO - sombra; ME - medicinal; * - Total de publicações com registro de uso dos componentes. 


\section{Espécies empregadas}

Dentro da base bibliográfica catalogada, há citação de cerca de 330 espécies, abrangendo os mais variados usos (Tabela 5). Dentre essas, as mais citadas são frutíferas: cupuaçu (Theobroma grandiflorum, 42\%) e pupunha (Bactris gasipaes, 33\%). Tal fato pode ser devido à ampla aceitação dessas duas espécies para consumo e comercialização. Dentre as espécies madeiráveis, as mais citadas foram: mogno (Swietenia macrophylla; 19\%) e paricá (Schyzolobium amazonicum; 16\%). Um destaque deve ser dado à castanha-do-pará (Bertholletia excelsa) com $32 \%$ das publicações indicando uma orientação das pesquisas em SAF para uma espécie nativa de uso múltiplo.

Tabela 5. Espécies vegetais citadas nas publicações de sistemas agroflorestais na Amazônia brasileira ao longo de série histórica (1980-2005).

\begin{tabular}{|c|c|c|}
\hline $\begin{array}{l}\text { Frequência } \\
\text { citações }\end{array}$ & $\begin{array}{l}\text { Número } \\
\text { espécies }\end{array}$ & Espécies \\
\hline $40-50 \%$ & 01 & Theobroma grandiflorum (42\%) \\
\hline $30-40 \%$ & 02 & Bactris gasipaes (33\%); Bertholletia excelsa $(32 \%)$ \\
\hline $20-30 \%$ & 02 & Musa sp. (21\%); Manihot esculenta (20\%) \\
\hline $15-20 \%$ & 06 & $\begin{array}{l}\text { Swietenia macrophylla }(19 \%) \text {; Euterpe oleracea }(18 \%) ; \text { Inga edulis }(18 \%) ; \text { Zea mays }(17 \%) \text {; } \\
\text { Schylozobium amazonicum }(16 \%) \text {; Hevea brasiliensis }(15 \%)\end{array}$ \\
\hline $10-15 \%$ & 05 & $\begin{array}{l}\text { Theobroma cacao (13\%); Gliricidia sepium }(12 \%) ; \text { Oryza sativa }(12 \%) \text {; Tectona grandis } \\
(11 \%) \text {; Bixa orellana }(10 \%)\end{array}$ \\
\hline $05-10 \%$ & 20 & 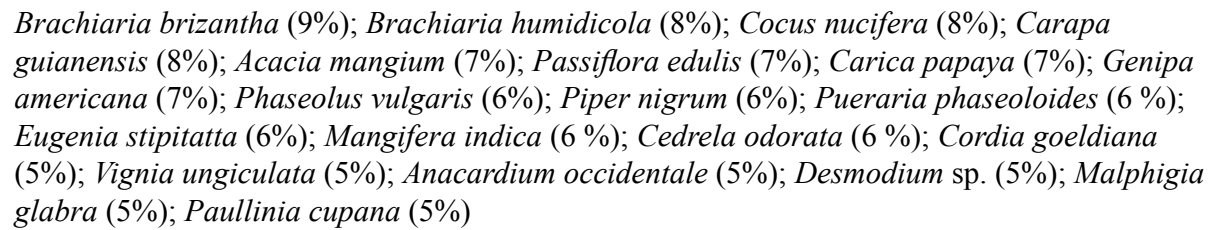 \\
\hline
\end{tabular}

Mais do que a frequência de citação de uso da espécie, é preciso levar em consideração a viabilidade de inserção do produto no mercado. Assim, ao fomentar futuros SAFs, é preciso pensar no mercado a ser atingido, considerando situação atual e projeções futuras. A título de exemplo, podem ser mencionados os casos do cacau (Theobroma cacao, $13 \%$ ) e da seringueira (Hevea brasiliensis, $15 \%$ ), que possuem um mercado demandante por sementes e látex.

De forma geral, os trabalhos publicados tratam de poucas espécies ao mesmo tempo. Em termos cumulativos, $60 \%$ da literatura usaram seis espécies (Figura 2). Entretanto, o número de trabalhos com 15 ou mais espécies foi cerca de $5 \%$. Considerando-se todas as publicações, em $90 \%$ dos trabalhos foram encontradas até 13 espécies em uma publicação, embora existam cinco trabalhos com mais 20 espécies (Figura 2). A constatação de poucos trabalhos com muitas espécies pode refletir a dificuldade de manejo destas em um único sistema de produção.

\section{Linhas de pesquisa}

$\mathrm{Na}$ série histórica estudada, $68 \%$ dos trabalhos publicados abordam uma única linha de pesquisa. $\mathrm{Na}$ década de 80 , essa frequência foi de $85 \%$, tendo uma redução na década de $90(65 \%)$ e um retorno ao patamar global até a metade da década de 2000 (68\%). Avaliandose as linhas de pesquisa, isoladamente, visualizam-se três grandes focos de estudos dos SAFs: componente vegetal, componente animal e outros indicadores (Figura 3). 


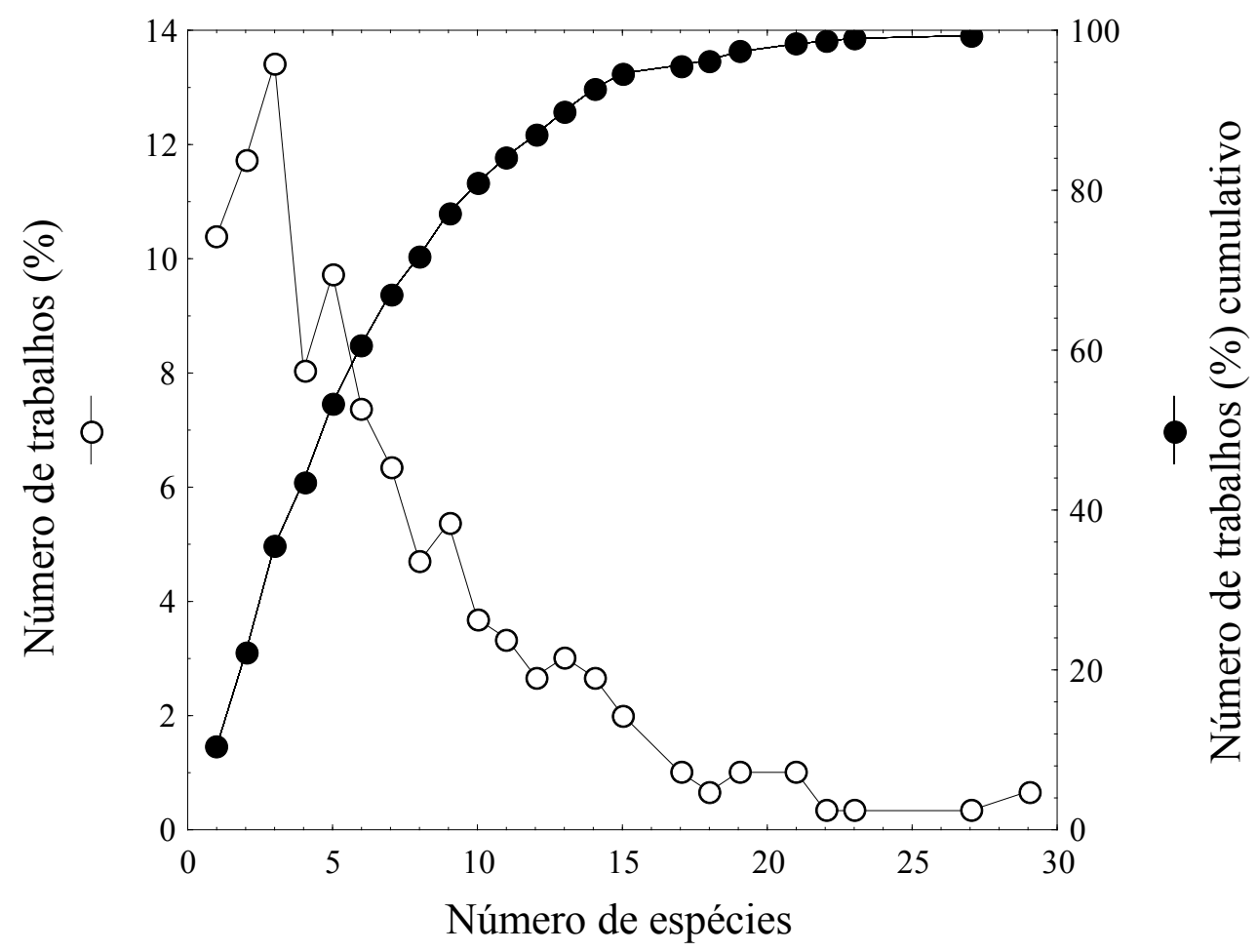

Figura 2. Frequência relativa de número de espécies listadas ou avaliadas em trabalhos sobre sistemas agroflorestais na Amazônia brasileira, ao longo de série histórica (1980-2005).

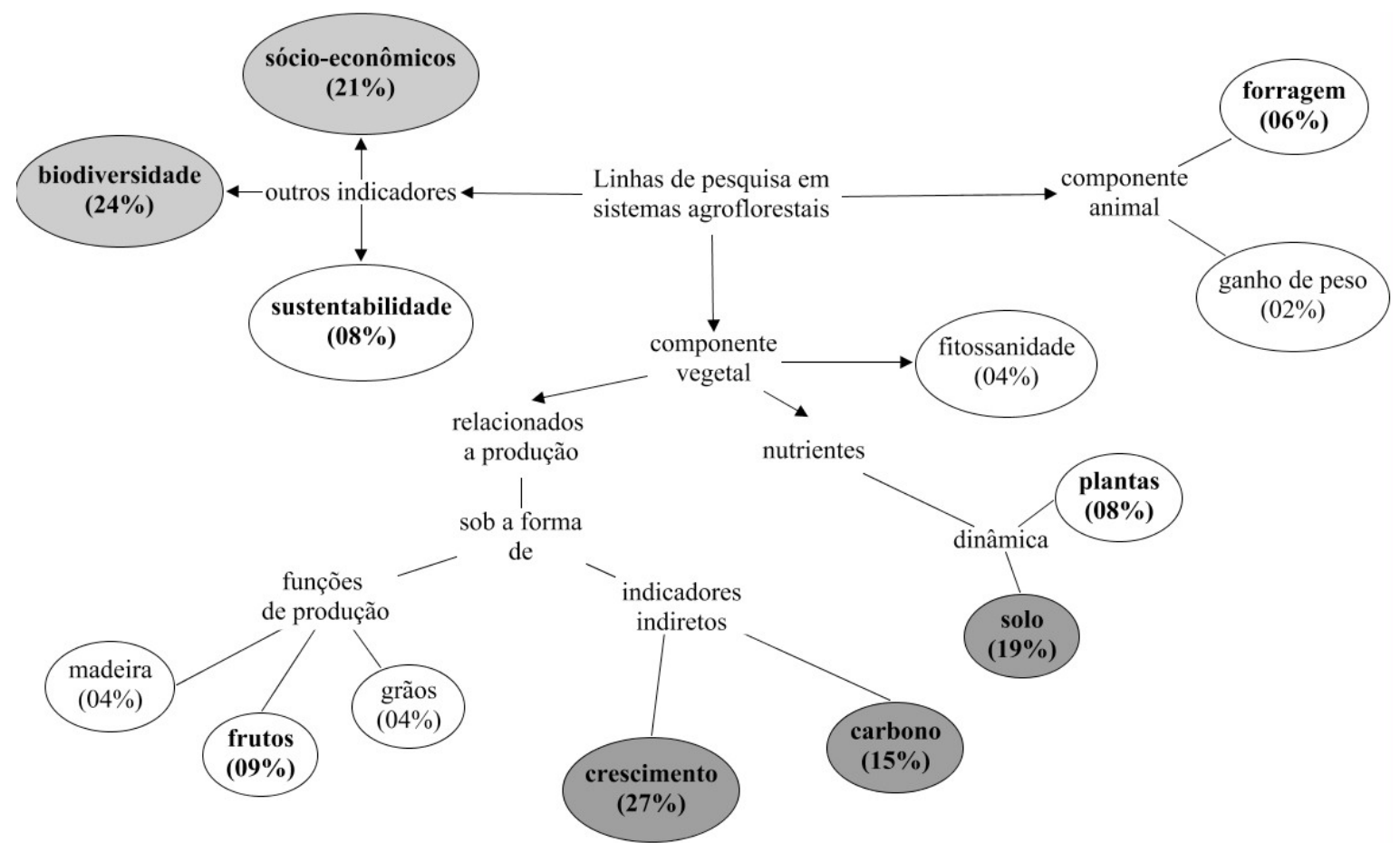

Figura 3. Tipologia das linhas de pesquisa em sistemas agroflorestais na Amazônia brasileira, ao longo de série histórica (1980-2005). Valores em negrito: menos de 5\% do total de publicações; Elipses preenchidas: mais de $10 \%$ do total de publicações. 
O componente vegetal mostrou ramificação mais intensa em termos de linhas de pesquisa, com três segmentações: (i) fitossanidade, (ii) dinâmica de nutrientes e (iii) produção vegetal. Apenas fitossanidade não apresentou uma segmentação hierárquica. No caso da dinâmica de nutrientes, percebe-se uma divisão quanto à alocação de nutrientes na planta ou no solo.

Nos trabalhos relacionados ao componente produção vegetal, houve concentração em função da natureza de indicadores: (a) diretos (produção de grãos, frutos e madeira); e (b) indiretos (crescimento e carbono). Neste componente, os segmentos mais representativos foram os estudos de dinâmica de nutrientes no solo (19\%) e os indicadores indiretos de produção: carbono $(15 \%)$ e crescimento $(27 \%)$.

O componente outros indicadores, constituído por pesquisas relacionadas aos aspectos socioeconômicos, sustentabilidade e estimação de diversidade, representou a grande maioria dos trabalhos (46\%). Destaque especial deve ser dado à estimação de biodiversidade (24\%), indicadores socioeconômicos (21\%) e, em menor escala, à sustentabilidade $(8 \%)$.

Comparado aos outros focos de estudos de SAFs já mencionados, o componente animal foi o que apresentou o menor número de publicações $(8 \%)$, abrangendo pesquisas relacionadas ao ganho de peso animal e acúmulo de biomassa de forrageiras. Deste modo, percebe-se que ainda são poucos os estudos com SAFs pecuários (sistemas agrossilvipastoris e silvipastoris), e apenas $6 \%$ dos trabalhos abordam aspectos biofísicos e o papel do componente agrícola nesses sistemas.

A atividade pecuária é representativa na paisagem amazônica (VEIGA; TOURRAND, 2002), entretanto, estudos de SAFs para este tipo de uso da terra são incipientes, logo, pesquisas futuras devem colocar mais atenção em SAFs pecuários, abordando: implantação do sistema, desempenho produtivo e reprodutivo animal, comportamento de forrageiras e desempenho silvicultural das árvores plantadas.

\section{Conclusões}

A produção científica sobre SAFs na Amazônia mostrou forte relação com os eventos científicos específicos à área agroflorestal, especialmente o Congresso Brasileiro de Sistemas Agroflorestais. A constatação de uma produção científica crescente indica condições para subsidiar periódicos dedicados ao tema.
Sistemas agroflorestais baseados em componentes alimentares foram os mais estudados na região, o que reflete uma preocupação com relação à segurança alimentar. Dentre as espécies mais estudadas tem-se: i) as alimentares Theobroma grandiflorum (cupuaçu), Bactris gasipaes (pupunha); ii) as madeiráveis Swietenia macrophylla (mogno) e Schyzolobium amazonicum (paricá); e iii) as de uso múltiplo como a Bertholletia excelsa (castanha-do-pará).

A pesquisa de SAFs em 25 anos pôde ser estruturada nos componentes vegetal, animal e outros indicadores do sistema. A topologia das linhas de pesquisas mostra que há uma concentração de trabalhos em: i) indicadores indiretos das funções de produção vegetal, tais como carbono e crescimento; e (ii) outros indicadores como biodiversidade e avaliações socioeconômicas. Um outro segmento de destaque do componente vegetal refere-se aos estudos de dinâmica de nutrientes no solo e na planta. $\mathrm{Na}$ Amazônia, embora a pecuária seja um representativo uso da terra, a pesquisa de SAFs pecuários é incipiente, havendo a necessidade de um fortalecimento de pesquisa nesta linha temática.

\section{Referências}

ALMEIDA, C. A. Estimativa da Área de Ocorrência e do Tempo de Permanência da Vegetação Secundária da Amazônia Legal com Imagens TM/Landsat. 2008. 129 p. (INPE--T/). Dissertação (Mestrado em Sensoriamento Remoto) - Instituto Nacional de Pesquisas Espaciais, São José dos Campos.

ARCO-VERDE, M. F; MOURÃO JÚNIOR, M.; LOPES, C. E. V; FREITAS, F. N. Diagnóstico sócio-econômico em áreas de pequenos produtores rurais no estado de Roraima, Brasil. Revista Acadêmica Ciências Agrárias e Ambientais, Curitiba, v. 2, n. 1, p. 61-64, 2004.

ÁVILA, M.; MINAE, S. Diagnosis and Design Methodology. In: TRIPATHL, B. R; PSYCHAS, P. J (ed). The AFNETA alley farming training manual - Volume 2: Source book for alley farming research. 1992. Disponível em: < http://www. ilri.org/InfoServ/Webpub/Fulldocs/X5546e/x5546e07. htm\#TopOfPage $>$. Acesso em: 06 jun. 2009.

BARRETO, P.; SOUZA JÚNIOR, C.; NOGUERÓN, R.; ANDERSON, A.; SALOMÃO, R. Human Pressure on the Brazilian Amazon Forests. WRI: Washington DC, Imazon: Belém. 2006. 84 p.

BRIENZA JÚNIOR, S. Biomass Dynamics of Fallow Vegetation Enriched with Leguminous Trees in the Eastern Amazon of Brazil. Georg-August: University of Gottingen, 1999. 133 p. Tese Doutorado. 
BRYANT, D.; NIELSEN, D.; TANGLEY, L. The last frontier forests: ecosystems and economies on the edge. World Resources Institute. Washington, D. C. 1997. Disponível em < http://pdf.wri.org/last_frontier_forests.pdf $>$ Acesso em: 5 abr. 2009.

CAÑAS, A. J.; HILL, G.; CARFF, R.; SURI, N.; LOTT, J.; ESKRIDGE, T.; GÓMEZ, G.; ARROYO, M.; CARVAJAL, R. CmapTools: A Knowledge Modeling and Sharing Environment, In: Concept Maps: Theory, Methodology, Technology, Proceedings of the First International Conference on Concept Mapping. CAÑAS A.J.; NOVAK J.D.; GONZÁLEZ, F.M., Editors. Universidad Pública de Navarra: Pamplona, Spain. 2004. p. 125-133.

COCHRANE, M.; SCHULZE, M. Forest fires in the Brazilian Amazon. Conservation Biology, v. 12, n. 5, p. 948-950, 1998 a.

COCHRANE, M.; SCHULZE, M. Fire as a recurrent event in tropical forests of the eastern Amazon: effects on forest structure, biomass, and species composition. Biotropica, v. 31, n. 1, p. 2-16, 1998b.

CRAVO, M. da S.; CORTELETTI, J.; NOGUEIRA, O. L.; SMYTH, T. J.; SOUZA, B. D. L. de. Sistema Bragantino: agricultura sustentável para a Amazônia. Belém, PA. Embrapa Amazônia Oriental. 2005. 93p. (Embrapa Amazônia Oriental. Documentos, 218).

DUBOIS, J. C. L.; VIANA, V. M.; ANDERSON, A. Manual agroflorestal para a Amazônia. Rio de Janeiro: REBRAF. 1996. $228 \mathrm{p}$.

\section{EMBRAPA AMAZÔNIA ORIENTAL. Pesquisa \&}

desenvolvimento: IAN-IPEAN-CPATU-Embrapa Amazônia

Oriental - há mais de seis décadas contribuindo para o

desenvolvimento sustentável da Amazônia. Belém, PA:

Embrapa Amazônia Oriental, 2003. 80 p. il.

INPE. Instituto Nacional de Pesquisa Espacial. 2009. São José dos Campos, São Paulo. Disponível em < http://www.dpi.inpe.br/ prodesdigital/prodesmunicipal.php>. Acesso em: 4 abr. 2009.

KAIMOWITZ, D.; ANGELSEN, A. Economic models of tropical deforestation: a review. Bogor, Indonesia. Center for International Forestry Research (CIFOR). 1998.

KATO, M. do S. A. Fire-free land preparation as an alternative to slash-and-burn agriculture in the bragantina region, eastern Amazon: crop performance and phosphorus dynamics. Gottingen: Cuvillier, 1998a.. 144p. il. Doctoral Dissertation.
$\mathrm{KATO}, \mathrm{O}$. R. Fire-free land preparation as an alternative to slash-and-burn agriculture in the bragantina region, eastern Amazon: crop performance and nitrogen dynamics. Gottingen: Cuvillier, 1998b. 132p. il. Doctoral Dissertation.

MANESCHY, R. Q.; SARMENTO, C. M. B.; BRIENZA JUNIOR, S.; YARED, J. A. G.; VEIGA, J. B. da. Uma análise sobre sistemas silvipastoris na Amazônia brasileira. In: CONGRESSO BRASILEIRO DE AGROECOLOGIA, 3.; SEMINÁRIO ESTADUAL DE AGROECOLOGIA, 3., 2005, Florianópolis. A sociedade construindo conhecimentos para a vida: anais. Florianópolis: Associação Brasileira de Agroecologia, 2005.

MOURA CARVALHO, L. O. D. de; COSTA, N. A. da. (Ed.). Sistemas de pastejo rotacionado intensivo manual técnico. Belém, PA: EMBRAPA-CPATU, 1998. 53 p. il.

SÁ, T. D. A. Meteorology applied to agroforestry systems in the Brazilian Amazon region. In: REIFSNYDER, W. S.; DARNHOFER, T. O. 1987. Meteorology and agroforestry: proceedings of an international workshop on the Application of Meteorology to Agroforestry Systems Planning and Management, Nairobi, 9-13 February 1987,Nairobi: ICRAF, 1989, p. 245-255.

SMITH, N.; DUBOIS, J.; CURRENT, D.; LUTZ, E.; CLEMENT, C. Experiências agroflorestais na Amazônia brasileira: restrições e oportunidades. Programa Piloto para a Proteção das Florestas Tropicais do Brasil. Brasília. 146 p.; il. 1998.

SORRENSEN, C. L. Linking smallholder land use and fire activity: examining biomass burning in the Brazilian Lower Amazon. Forest Ecology and Management, v. 128, n. 1-2, p. $11-25,2000$.

TRECENTI, R.; OLIVEIRA, M. C. de; HASS, G. 2008. Integração lavoura-pecuária-silvicultura. In.: TRECENTI, R.; OLIVEIRA, M. C. de; HASS, G. (eds). Integração lavourapecuária-silvicultura. Brasília: MAPA/SDC, 2008. 2-19 p.

VEIGA, J. B.; TOURRAND, J. F. Potencial e adoção de sistemas silvipastoris na Amazônia Oriental. In: MÜLLER, M. W.; GAMARODRIGUES, A. C.; BRANDÃO, I. C. F. L.; SERÔDIO, M. H. C. F. (Ed.) Sistemas agroflorestais, tendências da agricultura ecológica nos trópicos: sustento sustento da vida e sustento de vida. SBSAF/CEPLAC/UENF. Campos dos Goytacazes. 2004, p. 107-124.

VOSTI, S. A.; WITCOVER, J.; CARPENTIER, C. L. Agricultural intensification by smallholders in the western Brazilian Amazon: From deforestation to sustainable land use Washington,DC: International Food Policy Research Institute, 2002. 135 p. (Research report, 130)

Recebido em 07 de abril de 2009 e aprovado em 03 de dezembro de 2009 